\title{
O USO DO PLASMA RICO EM PLAQUETAS ASSOCIADO AO ASPIRADO DE MEDULAR ÓSSEA NA OSTEOTOMIA TIBIAL TIPO PUDDU
}

\author{
THE USE OF PLATELET RICH PLASMA ENRICHED WITH \\ BONE MARROW ASPIRATE IN PUDDU TIBIAL OSTEOTOMY
}

Caio Oliveira D'Elia', Márcia Uchoa de Rezende ${ }^{2}$, Alexandre Carneiro Bitar ${ }^{3}$, Nelson Tatsui ${ }^{4}$, José Ricardo Pécora ${ }^{5}$, Gilberto Luis Camanho ${ }^{6}$

\section{RESUMO}

Objetivo: O presente estudo procurou avaliar a aplicação do plasma rico em plaquetas associado ao aspirado de medular óssea como substituto ósseo ao enxerto autólogo do ilíaco nas osteotomias tibiais proximais de cunha de adição medial (OTCAM). Métodos: Foram estudados 25 pacientes submetidos a OTCAM divididos de forma randomizada em dois grupos, grupo ilíaco, 14 pacientes submetidos a OTCAM nos quais se utilizou o enxerto autólogo do ilíaco para preencher o sitio da osteotomia, grupo PRP, 11 pacientes submetidos a OTCAM nos quais se utilizou um composto formado por plasma rico em plaquetas associado ao aspirado de medular ósseo para preencher o sitio da osteotomia. Foram avaliados o sangramento (variação dos níveis de hemoglobina e hematócrito) e a dor (escala visual analógica -EVA), comparando os grupos em relação a essas variáveis. Resultados: Não foram observadas diferenças entre os grupos no que se refere à variação dos níveis de hemoglobina $(p=0,820)$ e hematócrito $(p=0,323)$. Os grupos não foram diferentes em relação à intensidade da dor segundo a EVA $(p=0,538)$. Conclusão: O uso do PRP associado ao aspirado de medular óssea nas OTCAM não demonstrou vantagem sobre a utilização do enxerto autólogo do ilíaco no que se refere a dor e sangramento.

Descritores - Plasma rico em plaquetas; Osteotomia; Tíbia; Substitutos ósseos

\section{ABSTRACT}

Objective: The present study was performed in order to evaluate the use of platelet rich plasma associated to bone marrow aspirate, substituting autologous iliac bone graft in medial opening wedge osteotomy (OWHTO). Methods: Twenty-five patients were submitted to tibial opening wedge osteotomy, being divided into two groups. Iliac group: 14 patients submitted to OWHTO, using autologous iliac bone graft to fill the gap. PRP group: 11 patients using platelet rich plasma associated to bone marrow aspirate to fill the gap. We evaluated bleeding (hemoglobin and hematocrit levels) and pain (visual analogic scale-VAS), then we compared the groups regarding these variables. Results: Differences between the groups were not found regarding hemoglobin levels $(p=0.820)$ and hematocrit levels $(p=0.323)$. The groups were not different regarding pain measured with VAS $(p=0.538)$. Conclusion: The use of platelet rich plasma associated to bone marrow aspirate in medial opening wedge osteotomy did not offer advantages over autologous iliac bone graft regarding bleeding and pain.

Keywords - Platelet rich plasma; Osteotomy; Tibia; Bone substitutes

\footnotetext{
1 - Pós-graduando do Programa de Pós-Graduação do Departamento de Ortopedia e Traumatologia da Faculdade de Medicina da USP.

2 - Médica Assistente do Grupo de Joelho do Instituto de Ortopedia e Traumatologia do HC/FMUSP.

3 - Médico Ortopedista do Instituto de Ortopedia e Traumatologia do HC/FMUSP.

4 - Médico Assistente do Departamento de Hematologia do HC/FMUSP.

5 - Chefe do Grupo do Joelho do Instituto de Ortopedia e Traumatologia do HC/FMUSP.

6 - Professor Associado da Faculdade de Medicina da Universidade de São Paulo.

Correspondência: Rua Dr. Ovídio Pires de Campos, 333 - São Paulo, SP. E-mail: caiodelia.vita@gmail.com
} 


\section{INTRODUÇÃO}

Plasma rico em plaquetas (PRP) refere-se a um preparado obtido a partir do sangue autólogo. As plaquetas possuem em seu interior grânulos repletos de fatores de crescimento, dentre os mais importantes: fator de crescimento derivado da plaqueta (PDGF), fator de crescimento transformador tipo beta (TGF- $\beta$ ) e fator endotelial de crescimento vascular $(\mathrm{VEGF})^{(1,2)}$.

Fatores de crescimento são polipeptídios, geralmente sintetizados por tecidos específicos, e que atuam como reguladores locais da função celular. Esses fatores de crescimento ligam-se a receptores de membrana na célula alvo, ativando um processo intracelular, que produzirá proteínas a serem utilizadas dentro da célula ou exportadas ${ }^{(3)}$.

A concentração normal de plaquetas no sangue periférico é em média de $150.000 / \mu 1$ a $350.000 / \mu 1$. Para que o PRP tenha maior eficácia, a concentração ideal de plaquetas deve ser em torno de $1.000 .000 / \mu 1^{(1,2,4)}$. Existem diversos métodos de obtenção de $\mathrm{PRP}^{(5)}$, cada um com sua peculiaridade em relação à capacidade de concentração das plaquetas, assim como no processo de liberação dos fatores de crescimento pelas plaquetas.

A utilização clínica do PRP vem crescendo consideravelmente, tendo sido aplicado em diversas situações: artroplastias de joelho (ATJ), reparos tendíneos, tratamento de lesões de cartilagem e como substituto ósseo ${ }^{(6-16)}$.

O PRP mostrou-se capaz de diminuir o sangramento, a dor e a ocorrência de artrofibrose quando aplicado no período perioperatório nas $\mathrm{ATJ}^{(6,7)}$.

O presente estudo tem como objetivo avaliar os resultados obtidos com a aplicação do PRP, no que se refere a dor e sangramento, nas osteotomias tibiais proximais por cunha de adição medial (OTCAM).

\section{MÉTODOS}

O presente estudo aprovado pelo Comitê de Ética em Pesquisa da Universidade de São Paulo. Todos os pacientes assinaram o termo de informação e consentimento.

Os critérios de inclusão no estudo foram: pacientes com deformidade em varo do joelho avaliada a partir de uma radiografia panorâmica com carga dos membros inferiores, pacientes entre 25 e 60 anos de idade, ausência de doenças inflamatórias sistêmicas (artrite reumatoide, lúpus, etc.), índice de massa corpórea (IMC) menor que $30 \mathrm{~kg} / \mathrm{m}^{2(17)}$, necessidade de correção com a utilização de cunhas entre $10 \mathrm{~mm}$ e $15 \mathrm{~mm}$. Diagnóstico de osteoartrose unicompartimental, deficiências ligamentares crô- nicas, ou deformidades dos membros inferiores. Foram respeitadas as indicações para osteotomia valgizante recomendadas na literatura ${ }^{(18-20)}$.

Os critérios de exclusão foram: perda de seguimento, solicitação do paciente para ser excluído do estudo.

Foram estudados 25 pacientes distribuídos em dois grupos, de forma randomizada, através de sorteio aleatório com reposição realizado no dia do agendamento do procedimento cirúrgico. Todos os procedimentos cirúrgicos neste estudo tiveram a participação do mesmo cirurgião.

Os pacientes foram então separados em dois grupos. O grupo ilíaco (GI) foi formado por 14 pacientes submetidos à osteotomia com a utilização de enxerto autólogo do ilíaco no sitio da osteotomia. O grupo PRP (GPRP) foi formado por 11 pacientes submetidos à osteotomia com utilização de um substituto ósseo composto por plasma rico em plaquetas (PRP) e aspirado de medular óssea. Denominamos esse substituto ósseo de enxerto ósseo biológico.

Em relação aos diagnósticos, os dois grupos apresentaram como o mais frequente a lesão crônica do ligamento cruzado anterior (LCA) (Tabela 1).

Tabela 1 - Distribuição dos pacientes dos grupos ilíaco e PRP segundo diagnóstico

\begin{tabular}{c|c|c|c|c}
\hline \multirow{2}{*}{} & & \multicolumn{2}{|c|}{ Grupos } & \multirow{2}{*}{ TOTAL } \\
\cline { 3 - 4 } & & llíaco & PRP & TOL \\
\hline \multirow{2}{*}{$\begin{array}{c}\text { Diagnóstico } \\
\mathbf{p}=\mathbf{0 , 1 6 8}\end{array}$} & \multirow{2}{*}{$\begin{array}{c}\text { Lesão crônica } \\
\text { do LCA }\end{array}$} & 6 & 9 & 15 \\
\cline { 3 - 5 } & \multirow{2}{*}{ OA medial $^{2}$} & $42,9 \%$ & $81,2 \%$ & $60,0 \%$ \\
\cline { 3 - 5 } & & 5 & 1 & 6 \\
\hline \multirow{2}{*}{ Total } & \multirow{2}{*}{ Deformidades $^{3}$} & 3 & $9,1 \%$ & $24,0 \%$ \\
\cline { 3 - 5 } & & $21,4 \%$ & $9,1 \%$ & $16,0 \%$ \\
\hline & & 14 & 11 & 25 \\
\hline & & $100 \%$ & $100 \%$ & $100 \%$ \\
\hline
\end{tabular}

Valor de $\mathrm{p}$ referente ao teste exato de Fisher

${ }^{1}$ Duplo varo ou triplo varo.

${ }^{2}$ Inclui também osteonecrose.

${ }^{3}$ Genuvaro ou sequela de fratura.

Os grupos foram homogêneos no que se refere ao tamanho das cunhas utilizadas (Tabela 2).

A idade média dos pacientes dos grupos ilíaco e PRP foi de 45,9 e 37,8 anos, respectivamente ( $p=0,014)$.

A técnica empregada para a realização da osteotomia foi, como já referido anteriormente, a adição de cunha medial, à semelhança do planejamento e técnica cirúrgica descritos por Puddu et al ${ }^{(19,20)}$. 
Tabela 2 - Distribuição dos pacientes dos grupos ilíaco e PRP segundo tamanho da cunha utilizada

\begin{tabular}{|c|c|c|c|c|}
\hline & & \multicolumn{2}{|c|}{ Grupos } & \multirow{2}{*}{ TOTAL } \\
\hline & & llíaco & PRP & \\
\hline \multirow{4}{*}{$\begin{array}{l}\text { Tamanho } \\
\text { da cunha } \\
p=0,885\end{array}$} & \multirow{2}{*}{$10,0 \mathrm{~mm}$} & 5 & 3 & 8 \\
\hline & & $35,7 \%$ & $27,3 \%$ & $32,0 \%$ \\
\hline & \multirow{2}{*}{$12,5 \mathrm{~mm}$} & 5 & 5 & 10 \\
\hline & & $35,7 \%$ & $45,5 \%$ & $40,0 \%$ \\
\hline & \multirow{2}{*}{$15,0 \mathrm{~mm}$} & 4 & 3 & 7 \\
\hline & & $28,6 \%$ & $27,3 \%$ & $28,0 \%$ \\
\hline \multirow{2}{*}{ TOTAL } & & 14 & 11 & 25 \\
\hline & & $100 \%$ & $100 \%$ & $100 \%$ \\
\hline
\end{tabular}

Valor de $\mathrm{p}$ referente ao teste exato de Fisher

Após a realização da osteotomia e fixação da placa, o espaço criado na região metafisária da tíbia era preenchido por um dos enxertos avaliados (ilíaco ou biológico).

Para coleta das plaquetas utilizamos o separador celular automático Haemonetics MCS+ 9000 e o kit específico para plaquetaférese 995-E (Haemonetics Corp.). Nesse sistema, por meio de uma punção venosa em fossa antecubital, o sangue do próprio paciente era drenado para um dispositivo de separação sob centrifugação contínua (Figura 1). Após o fracionamento do sangue, um analisador de refração ótica individualizava a camada plaquetária e determinava a sua coleta em bolsa descartável específica. O sangue remanescente era totalmente reinfundido no paciente, determinando o fim de um ciclo. Citrato de sódio foi usado como anticoagulante na proporção de um para cada $9 \mathrm{ml} \mathrm{de}$ sangue total processado. Habitualmente, realizávamos dois ciclos, coletando aproximadamente $70 \mathrm{ml}$ de concentrado de plaquetas.

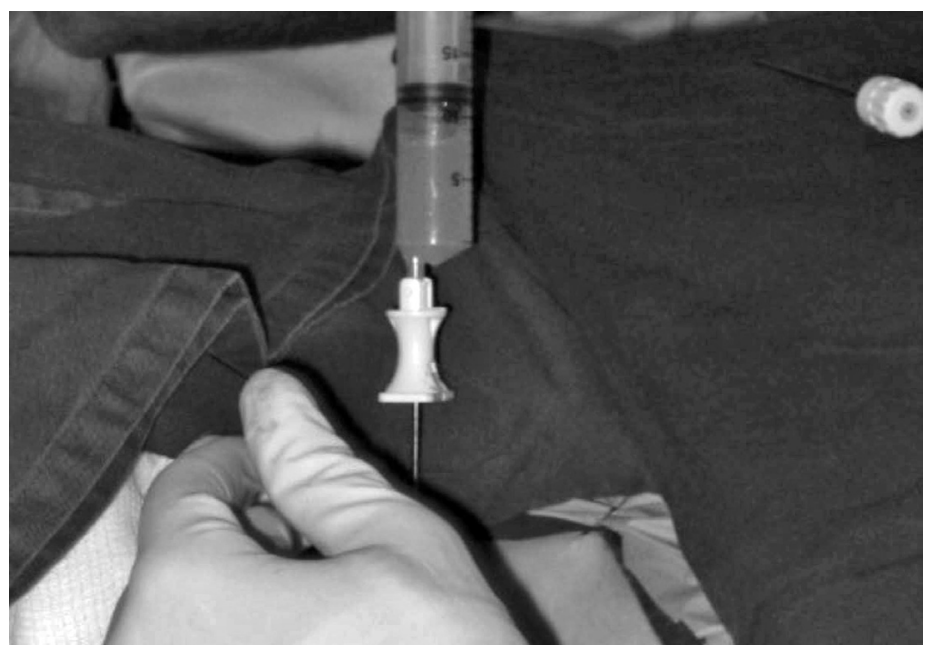

Figura 2 - Obtenção do aspirado de medular óssea

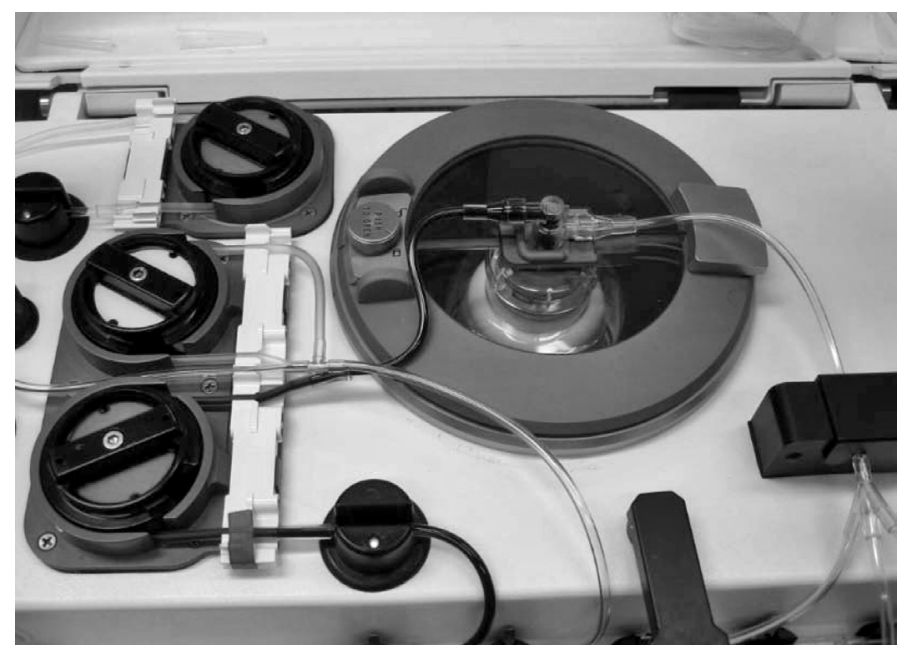

Figura 1 - Separador celular automático Haemonetics MCS +9000

O aspirado de medula óssea foi obtido da crista ilíaca através de punção percutânea, utilizando técnica padronizada (Figura 2). Realizavam-se seis punções para obter cerca de $12 \mathrm{ml}$ de medula óssea, de forma a não exceder o volume de $2 \mathrm{ml}$ por punção; após cada punção, a agulha era reposicionada ${ }^{(21)}$. Esse material era anticoagulado com citrato de sódio na proporção citrato:medular-óssea de 1:5.

O enxerto ósseo biológico era formado pela adição de aspirado de medular óssea ao concentrado de plaquetas. Uma vez formado o gel, o cirurgião procedia à colocação do material no sítio cirúrgico (Figura 3).

Para a avaliação do sangramento oriundo do procedimento cirúrgico foram obtidos os índices hematimétricos: níveis de hemoglobina $(\mathrm{Hb})$ e hematócrito $(\mathrm{Ht})^{(7)}$. Esses índices foram obtidos no dia do procedimento cirúrgico e 24 horas após a realização do mesmo. Obteve-

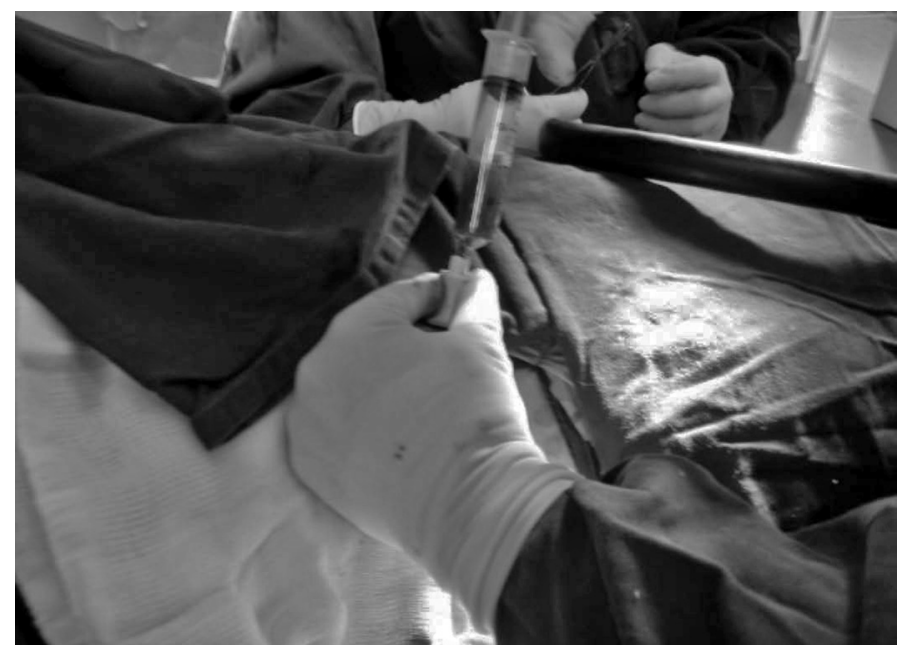




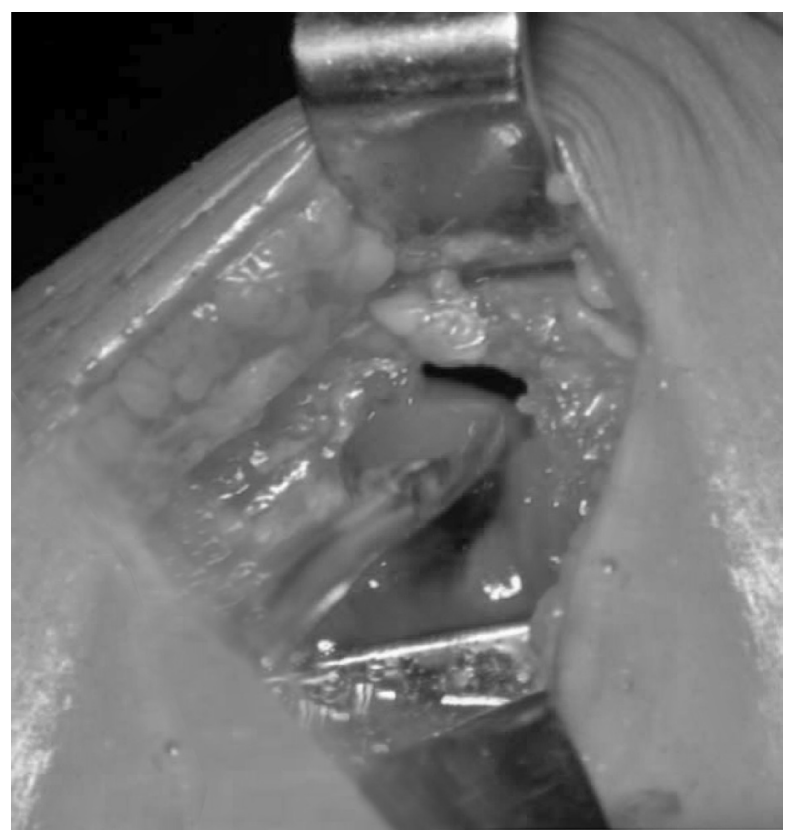

Figura 3 - Colocação do enxerto biológico no sítio da osteotomia

se então a variação pré e pós-operatória desses índices. Os grupos foram comparados entre si em relação a essas variáveis (variação de hemoglobina e hematócrito) pelo teste $t$ de Student.

A avaliação da dor foi feita através da escala visual analógica de dor ${ }^{(22,23)}$, realizada 24 horas após a cirurgia. Os grupos foram comparados entre si em relação a essa variável pelo teste $t$ de Student.

\section{RESULTADOS}

Não se observaram diferenças entre os grupos ilíaco e PRP no que se refere à variação dos níveis de hemoglobina e hematócrito pré e pós- operatórios (Tabelas 3 e 4).

A avaliação da dor feita através da escala visual analógica de dor (EVA) não foi diferente entre os grupos (Tabela 5).

Tabela 3 - Variação nos níveis de hemoglobina $(\mathrm{Hb})$

\begin{tabular}{c|l|c|c|c}
\hline & & $\begin{array}{c}\text { Ilíaco } \\
(\mathbf{n}=14)\end{array}$ & $\begin{array}{c}\text { PRP } \\
(\mathbf{n}=11)\end{array}$ & Total \\
\hline Diferença entre $\mathrm{Hb}$ & Média & 2,3 & 2,2 & 2,3 \\
\hline pré e pós-cirurgia & DP & 1,0 & 0,7 & 0,9 \\
\hline $\mathbf{p = 0 , 8 2 0}$ & Mediana & 2,4 & 2,1 & 2,2 \\
\hline & Mínimo & $-0,3$ & 0,9 & $-0,3$ \\
\hline & Máximo & 3,7 & 3,2 & 3,7 \\
\hline
\end{tabular}

Valor de $\mathrm{p}$ referente ao teste $t$ de Student.
Tabela 4 - Variação nos níveis do hematócrito $(\mathrm{Ht})$

\begin{tabular}{c|l|c|c|c}
\hline & & $\begin{array}{c}\text { llíaco } \\
(\mathbf{n}=14)\end{array}$ & $\begin{array}{c}\text { PRP } \\
(\mathbf{n}=11)\end{array}$ & Total \\
\hline Diferença entre Ht & Média & 6,4 & 5,3 & 5,9 \\
\hline pré e pós-cirurgia & DP & 2,3 & 3,1 & 2,7 \\
\hline $\mathbf{p = 0 , 3 2 3}$ & Mediana & 5,9 & 5,7 & 5,7 \\
\hline & Mínimo & 2,3 & 0,3 & 0,3 \\
\hline & Máximo & 10,8 & 9,2 & 10,8 \\
\hline
\end{tabular}

Valor de $\mathrm{p}$ referente ao teste $t$ de Student.

Tabela 5 - Escala visual analógica de dor ${ }^{(22)}$

\begin{tabular}{c|l|c|c|c}
\hline & & $\begin{array}{c}\text { Ilíaco } \\
(\mathbf{n}=14)\end{array}$ & $\begin{array}{c}\text { PRP } \\
(\mathbf{n}=11)\end{array}$ & Total \\
\hline Escala de dor & Média & 5,1 & 4,4 & 4,8 \\
\hline $\mathbf{p = 0 , 5 3 8}$ & DP & 2,9 & 2,7 & 2,8 \\
\hline & Mediana & 6,0 & 3,0 & 5,0 \\
\hline & Mínimo & 1,0 & 1,0 & 1,0 \\
\hline & Máximo & 9,0 & 9,0 & 9,0 \\
\hline
\end{tabular}

Valor de $\mathrm{p}$ referente ao teste $t$ de Student.

\section{DISCUSSÃO}

A osteotomia valgizante de tíbia empregando a técnica de adição de cunha medial é um procedimento frequente no tratamento de diversas patologias ortopédicas, sendo sua realização bem padronizada nos dias de hoje ${ }^{(18,24-27)}$.

O plasma rico em plaquetas (PRP) possui importante ação osteoindutora, como demonstrado por diversos estudos experimentais ${ }^{(11,28-31)}$. O PRP tem larga aplicação clínica na área de cirurgia bucomaxilofacial, sendo utilizado como agente osteopromotor em diversas situações $^{(31-33)}$. A sua utilização clínica em ortopedia vem crescendo, a despeito da ausência de estudos prospectivos e randomizados que avaliem os resultados de sua aplicação ${ }^{(30,34-36)}$.

Em um estudo que se propõe a avaliar a eficácia do PRP isolado ou em associação com outros materiais, é importante lembrar que diversos são os métodos utilizados na obtenção do $\operatorname{PRP}^{(1,5,30)}$, e que a capacidade em obter altas concentrações de plaquetas é variável entre os métodos disponíveis. A maioria dos sistemas utilizados na prática clínica é baseada na centrifugação; as centrífugas utilizadas para esse fim foram desenvolvidas para diagnóstico e não para a obtenção de PRP, que é gerado frequentemente com nível de plaquetas inferior ao ideal. O método de obtenção de PRP utilizado em nosso estudo ${ }^{(37)}$ é capaz de oferecer concentração de plaquetas superior a $1.000 .000 / \mu 1$. 
A necessidade da utilização do enxerto autólogo nas OTCAM é considerada uma desvantagem por muitos cirurgiões, em razão da morbidade associada à obtenção do enxerto autólogo ${ }^{(38,39)}$. A dor é uma queixa frequente nos pacientes que são submetidos à obtenção do enxerto autólogo. Em nosso estudo não verificamos diferença na variável dor após 24 horas entre os grupos $(\mathrm{p}=0,538)$.

O PRP foi utilizado nas ATJ ${ }^{(6,7)}$, diminuindo o sangramento e a ocorrência de artrofibrose. Em nosso modelo de aplicação clínica, a osteotomia tibial proximal por cunha de adição medial, não verificamos diferença no sangramento entre os grupos com a utilização do PRP associado ao aspirado de medular óssea $(\mathrm{p}=0,820$ e $\mathrm{p}=0,323)$.

\section{CONCLUSÃO}

$\mathrm{O}$ uso do PRP associado à medular óssea nas OTCAM não demonstrou vantagens sobre a utilização do enxerto autólogo do ilíaco no que se refere a dor e sangramento.

\section{REFERÊNCIAS}

1. Marx RE. Platelet-rich plasma (PRP): what is PRP and what is not PRP? Implant Dent. 2001;10(4):225-8.

2. Marx RE. Platelet-rich plasma: evidence to support its use. J Oral Maxillofac Surg. 2004;62(4):489-96.

3. Lind M. Growth factors: possible new clinical tools. A review. Acta Orthop Scand 1996;67(4):407-17.

4. Eppley BL, Woodell JE, Higgins J. Platelet quantification and growth factor analysis from platelet-rich plasma: implications for wound healing. Plast Reconstr Surg. 2004;114(6):1502-8.

5. Everts PA, Brown Mahoney C, Hoffmann JJ, Schonberger JP, Box HA, van Zundert A, et al. Platelet-rich plasma preparation using three devices: implications for platelet activation and platelet growth factor release. Growth Factors 2006;24(3):165-71.

6. Everts PA, Devilee RJ, Brown Mahoney C, Eeftinck-Schattenkerk M, Box HA Knape JT, et al. Platelet gel and fibrin sealant reduce allogeneic blood transfusions in total knee arthroplasty. Acta Anaesthesiol Scand. 2006;50(5):593-9.

7. Everts PA, Devilee RJ, Oosterbos CJ, Mahoney CB, Schattenkerk ME, Knape JT, et al. Autologous platelet gel and fibrin sealant enhance the efficacy of total knee arthroplasty: improved range of motion, decreased length of stay and a reduced incidence of arthrofibrosis. Knee Surg Sports Traumatol Arthrosc. 2007;15(7):888-94.

8. Sanchez M, Azofra J, Anitua E, Andia I, Padilla S, Santisteban J, et al. Plasma rich in growth factors to treat an articular cartilage avulsion: a case report. Med Sci Sports Exerc. 2003;35(10):1648-52.

9. Sanchez M, Anitua E, Azofra J, Andia I, Padilla S, Mujika I. Comparison of surgically repaired Achilles tendon tears using platelet-rich fibrin matrices. Am J Sports Med. 2007;35(2):245-51.

10. Lowery GL, Kulkarni S, Pennisi AE. Use of autologous growth factors in lumbar spinal fusion. Bone. 1999;25(2 Suppl):47S-50S.

11. Gandhi A, Doumas C, O'Connor JP, Parsons JR, Lin SS. The effects of local platelet rich plasma delivery on diabetic fracture healing. Bone. 2006;38(4):540-6.

12. Filho Cerruti H, Kerkis I, Kerkis A, Tatsui NH, da Costa Neves A, Bueno DF, et al. Allogenous bone grafts improved by bone marrow stem cells and platelet growth factors: clinical case reports. Artif Organs. 2007;31(4):268-73.

13. Choi BH, Im CJ, Huh JY, Suh JJ, Lee SH. Effect of platelet-rich plasma on bone regeneration in autogenous bone graft. Int J Oral Maxillofac Surg. 2004;33(1):56-9.

14. Bielecki T, Gazdzik TS, Szczepanski T. Benefit of percutaneous injection of autologous platelet-leukocyte-rich gel in patients with delayed union and nonunion. Eur Surg Res. 2008;40(3):289-96.

15. Bielecki T, Gazdzik TS, Szczepanski T. Re: "The effects of local platelet rich plasma delivery on diabetic fracture healing". What do we use: Platelet-rich plasma or platelet-rich gel? Bone. 2006;39(6):1388.

16. Dallari D, Savarino L, Stagni C, Cenni E, Cenacchi A, Fornasari PM, et al. Enhanced tibial osteotomy healing with use of bone grafts supplemented with platelet gel or platelet gel and bone marrow stromal cells. J Bone Joint Surg Am. 2007;89(11):2413-20.

17. Romero-Corral A, Somers VK, Sierra-Johnson J, Thomas RJ, Collazo-Clavell $\mathrm{ML}$, Korinek J, et al. Accuracy of body mass index in diagnosing obesity in the adult general population. Int J Obes (Lond). 2008;32(6):959-66.

18. Asik M, Sen C, Kilic B, Goksan SB, Ciftci F, Taser OF. High tibial osteotomy with Puddu plate for the treatment of varus gonarthrosis. Knee Surg Sports Traumatol Arthrosc. 2006;14(10):948-54.

19. Franco V, Cerullo G, Cipolla M, Gianni E, Puddu G. Open wedge high tibial osteotomy. Tech Knee Surg. 2002;1(1):43-53.
20. Puddu G, Cipolla M, Cerullo G, Franco V, Gianni E. Osteotomies: the surgical treatment of the valgus knee. Sports Med Arthrosc. 2007;15(1):15-22.

21. Muschler GF, Boehm C, Easley K. Aspiration to obtain osteoblast progenitor cells from human bone marrow: the influence of aspiration volume. J Bone Joint Surg Am. 1997;79(11):1699-709.

22. Willestaedt H, Levander G, Hult L. Studies in osteogenesis. Acta Orthop Scand. 1950;19(4):419-32.

23. DeLoach LJ, Higgins MS, Caplan AB, Stiff JL. The visual analog scale in the immediate postoperative period: intrasubject variability and correlation with a numeric scale. Anesth Analg. 1998;86(1):102-6.

24. Amendola A, Panarella L. High tibial osteotomy for the treatment of unicompartmental arthritis of the knee. Orthop Clin North Am. 2005;36(4):497-504.

25. Amendola A, Fowler PJ, Litchfield R, Kirkley S, Clatworthy M. Opening wedge high tibial osteotomy using a novel technique: early results and complications. J Knee Surg. 2004;17(3):164-9.

26. Brinkman JM, Lobenhoffer P, Agneskirchner JD, Staubli AE, Wymenga AB, van Heerwaarden RJ. Osteotomies around the knee: patient selection, stability of fixation and bone healing in high tibial osteotomies. J Bone Joint Surg Br. 2008;90(12):1548-57.

27. Koshino T, Murase T, Saito T. Medial opening-wedge high tibial osteotomy with use of porous hydroxyapatite to treat medial compartment osteoarthritis of the knee. J Bone Joint Surg Am. 2003;85-A(1):78-85.

28. Nash TJ, Howlett CR, Martin C, Steele J, Johnson KA, Hicklin DJ. Effect of plateletderived growth factor on tibial osteotomies in rabbits. Bone. 1994;15(2):203-8.

29. Anitua E, Andia I, Ardanza B, Nurden P, Nurden AT. Autologous platelets as a source of proteins for healing and tissue regeneration. Thromb Haemost. 2004;91(1):4-15.

30. Floryan KM, Berghoff WJ. Intraoperative use of autologous platelet-rich and platelet-poor plasma for orthopedic surgery patients. AORN J. 2004;80(4):668-74

31. Grageda E. Platelet-rich plasma and bone graft materials: a review and a standardized research protocol. Implant Dent. 2004;13(4):301-9.

32. Marx RE, Carlson ER, Eichstaedt RM, Schimmele SR, Strauss JE, Georgeff KR. Platelet-rich plasma: Growth factor enhancement for bone grafts. Oral Surg Oral Med Oral Pathol Oral Radiol Endod. 1998;85(6):638-46.

33. Sanchez AR, Sheridan PJ, Kupp LI. Is platelet-rich plasma the perfect enhancement factor? A current review. Int J Oral Maxillofac Implants. 2003;18(1):93-103.

34. Roldan JC, Jepsen S, Miller J, Freitag S, Rueger DC, Acil Y, et al. Bone formation in the presence of platelet-rich plasma vs. bone morphogenetic protein-7. Bone. 2004;34(1):80-90.

35. Solheim E. Growth factors in bone. Int Orthop. 1998;22(6):410-6.

36. Mariconda M, Cozzolino F, Cozzolino A, D’Agostino E, Bove A, Milano C. Platelet gel supplementation in long bone nonunions treated by external fixation. J Orthop Trauma. 2008;22(5):342-5.

37. O'Neill EM, Zalewski WM, Eaton LJ, Popovsky MA, Pivacek LE, Ragno G, et al. Autologous platelet-rich plasma isolated using the Haemonetics Cell Saver 5 and $\mathrm{Ha}$ emonetics MCS+ for the preparation of platelet gel. Vox Sang. 2001;81(3):172-5.

38. Warden SJ, Morris HG, Crossley KM, Brukner PD, Bennell KL. Delayed- and non-union following opening wedge high tibial osteotomy: surgeons' results from 182 completed cases. Knee Surg Sports Traumatol Arthrosc. 2005;13(1):34-7.

39. Spahn G. Complications in high tibial (medial opening wedge) osteotomy. Arch Orthop Trauma Surg. 2004;124(10):649-53. 